\title{
Short- And Long-Term Value-At-Risk, Skewness, Kurtosis and Coherent Risk Measure
}

\author{
Weiping $\mathrm{Li}^{1}$, Guotai $\mathrm{Chi}^{2} \&$ Bin Meng ${ }^{2}$ \\ ${ }^{1}$ Institute of Finance and Big Data, Southwest Jiaotong University, Chengdu, China and The Spears School of Business, \\ Oklahoma State University, Stillwater, OK, USA \\ ${ }^{2}$ Faculty of management and economics, Dalian University of Technology, Dalian, China \\ Correspondence: Weiping Li, Institute of Finance and Big Data, Southwest Jiaotong University, Chengdu, China and \\ The Spears School of Business, Oklahoma State University, Stillwater, OK, USA
}

Received: March 11, 2016

doi:10.11114/aef.v3i3.1528
Accepted: April 5, 2016

Available online: April 7, 2016

URL: http://dx.doi.org/10.11114/aef.v3i3.1528

\begin{abstract}
Standard risk management focuses on short-run risks rather than longer periods. We introduce an improved risk measure which can be used to estimate both short-and long-term structure of value at risk and the corresponding expected shortfall. The short- and long-term coherent measure of risk is specified and computed for both S\&P 500, HSI and SHSZ 300. We also test long-term skewness and kurtosis from empirical analysis for S\&P 500, HSI and SHSZ 300. We also show that our improved risk measure gives a better estimate of the value at risk for short horizons and never decreases to negative values like VaR for long-run horizons. Both long-term skewness and kurtosis for HSI and SHSZ 300 are analyzed empirically.
\end{abstract}

Keywords: risk management, short-term risk, long-term risk, skewness, kurotosis, systemic risk

\section{Introducation}

Engle (2011) raises an important challenge question on the risk assessment to measure both short- and long-term risk. The short-term nature of VaR and ES (expected loss) is a very important feature. Positions held for more than one day will have additional risk due to the fact that risk itself can change with respect to the time horizon (see Engle (2009)). Most investors and financial firms hold much longer than one day and changes in risk will be a very important determinant of returns. The risk measure VaR with specified quantile can be negative for long horizons ${ }^{1}$, where $P_{t}\left[S_{t+T} / S_{t}-1<-V a R_{\alpha, T}\right]=\alpha$ defines $\operatorname{VaR}_{\alpha, T}$ with the pre-determined quantile $\alpha$ and the value $S_{t}$ of the portfolio at $t$. Hence, the simple approximation of $\mathrm{VaR}$ by $\operatorname{VaR}_{0, T}-\mu T$ can no longer be used in the long-term risk measurement, where $\operatorname{VaR}_{\alpha, T}^{0}$ is defined by $P_{t}\left[s_{t+T}-E_{t} s_{t+T}<\ln \left(1-V a R_{\alpha, T}^{0}\right)\right]=\alpha$ and $s_{t}=\ln S_{t}$. Indeed the Securities Exchange Commission (SEC) tied the capital requirements of financial service firms to the losses in 1980 that would incurred with $95 \%$ confidence over 30 days interval in different security classes as the first regulation on VaR.

During the 2007-2008 financial crisis, many risk management systems fail to accurately assess the risks of financial positions and economic agents and financial firm managers fail to respond these risk appropriately. Acharya et al (2010) find these are two key features among other things. Engle (2011) points out that there two features are inextricably linked, and it is unlikely that we will be able to quantify the relative importance of miss-measurement of risk from the incentives to ignore risk. The most widely used risk measure with frequently criticized is the value at risk VaR of a firm or portfolio. This is the $1 \%$ quantile of the distribution of future values and is typically defined over the next trading day. The mis-use of the short-run VaR into assessing long-term risk during the financial crisis is arguably one of many causes for the 2007-2008 financial crisis.

\footnotetext{
${ }^{1}$ VaR focuses clearly on downside risk and potential loses. The demise of Long Term Capital Management, the investment fund with top pedigree Wall street traders and Noble Prize winners was a trigger to accept VaR largely.
} 
In this paper, we introduce an extension $\operatorname{VaR}$ of the traditional value at risk VaR for any quantile $\alpha$ level. Our generalized value at risk is sufficiently close to the variation of $\mathrm{VaR}$ for short time horizons, and is always positive for long-term horizons. Engle (2011) uses $V a R_{\alpha, T} \cong V a R_{\alpha, T}^{0}-\mu T$ as an approximation. The value at risk VaR evaluated by $\operatorname{VaR}_{\alpha, T}^{0}-\mu T$ will be negative after some long-term horizon $T_{0}\left(T>T_{0}\right.$ for a solution $T_{0}$ of the nonlinear equation $\mu T_{0}=\operatorname{VaR}_{\alpha, T_{0}}^{0}$ ). We study this property for both S\&P 500, HIS and SHSZ 300 to indicate that this is always the case. For these three indices, Engle's (2011) approximated VaR always decreases to negative value and our extension VaR always takes positive values and gradually increases to 1 (therefore loss everything for long-term horizons with this risk measure). In a certain sense, our extension $V a R$ of the value at risk VaR provides a risk measure for both short- and long-term risk and refines the value at risk $\operatorname{VaR}_{\alpha, T}^{0}$. Moreover, we show that $\operatorname{VaR}_{\alpha, T}^{0}$ can be thought of a linear approximation of $\operatorname{VaR}_{\alpha, T}^{0}$ in terms of $T$, and our extension $\operatorname{VaR}_{\alpha, T}$ can be thought of a quadratic (nonlinear) approximation of $\operatorname{VaR}_{\alpha, T}$ in terms of $T$.

J. P. Morgan in RiskMetrics system documentation states that Value at Risk is an estimate, with a predefined confidence interval, of how much one can lose from holding a position over a set horizon. RiskMetrics makes the variances in and covariances across asset classes freely available to any investor who wants to access them and to compute the Value at Risk analytically for a portfolio. Potential horizons may be one day for typical trading activities or a month or longer for portfolio management. VaR is typically measured from volatility or correlation models that are adjusted frequently to reflect changes in risk, a generic measure of risk exposure. VaR is a statistic measure of risk and an unconditional measure of risk which is also difficult to estimate. Engle (2011) develops a test to understand the strength of the long-term negative skewness as a property of asymmetric volatility models. He shows that asymmetric models used in Engle (2011) are consistent with the long-term skewness in the data. In this paper, we do not test those asymmetric models. Instead, we evaluate empirically the extended value at risk $V a R$ to the Hong Kong Hang Seng Index and the Chinese Hushen 300 index (SHSZ 300) from 1990 to 2012 and 2002 to 2012 respectively. Our extended VaR shows the sufficiently close to VaR evaluated in Engle (2011) for very short horizon in terms of $\operatorname{VaR}_{\alpha, T}^{0}$, and provide a better risk measure in the short horizon from the definition. Furthermore, $V a R$ decreases first and tips up to stay in positive range and approach to the maximum probability one.

Dubil (2009) shows that the focus on distribution simplifications, linear options approximations, variance modeling and heteroskedasticity does not help the VaR practitioner deal with the efficient loss value sampling faced by a bank with thousands of positions, hundreds of risk factors, various derivatives with nonlinear cross-termed factor exposure ${ }^{2}$. We use the normal distribution to estimate the extended VaR, and leave the further volatility roll-over (those five models studied in Engle (2011)) into the computation for a future study. From our empirical test on VaR, the extended VaR is more accurate in a short horizon, say 1-year. Hence, it would be possible to apply $V a R$ into Solvency II, QIS, Market Risk and Insurancerisks (see Egidio dos Rels et al (2009)). Jackson et al (1997) analyze the empirical performanceof different VaR models using data on the actual fixed income, foreign exchange andequity security holdings of a large bank.

Artzner et al $(1997,1999)$ define a unified frame work for market risks as well as nonmarketrisks without assuming completeness of markets. These coherent measures of risk satisfythe basic four properties (translation invariance, subadditivity, positive homogeneity and monotonicity) and can be adapted as capital requirements to regulate the risk. Acerbi and Tasche (2002) show that the expected shortfall is a coherent risk measure which resolvesthe deficiencies of value-at-risk. With our extended short- and long-term value-at-risk, we introduce the short- and long-term coherent measure of risk corresponding to the extended value-at-risk. The tail conditional expectation at a level $\alpha$ quantile, and the extended $\alpha$-tail mean as well as the extended expected shortfall are defined and computed in Section 3 . The extended expected shortfall is a coherent risk measure in the sense of Artzner et al (1999) and Delbaen (2002).

The paper is organized as follows. Section 2 introduces the extension of the value at risk defined by Engle (2011) and

\footnotetext{
${ }^{2}$ One should focus on the size relative to the standard deviation, i.e., a large (positive or negative) return in a period of high volatility may lead in a low standardized return, while the same rest urn following a period of low volatility may yield an abnormally high standardized return. Hence, the emphasis on normalized standardized return exposed the VaR computation to the risk of more frequent large outliers than would be expected with a normal distribution.
} 
studies its basic properties. Section 3 defines the extended tail conditional expectation, the extended $\alpha$-tail mean and the extended expected shortfall. Section 4 compares both our extension risk measure and VaR for short- and long-term horizons for S\&P 500, and the short- and long-term skewness and kurtosis of S\&P 500 are also presented. Section 5 illustrates the HSI and SHSZ 300 for both extended VaR and VaR as well as short- and long-term skewness and kurtosis. We conclude in Section 6.

\section{Short- and Long-term Structure of Risk}

The short- and long-term risks are measured from short horizons as one day or one week to long horizons as one year or many years. Usually the risk measure is considered as the losses that would result from underlying asset moves over various time horizons. The important challenge to risk assessment is to develop risk measures that can capture both short- and long-term risks, as Engle (2011) points out. This risk measure is the crucial instrument to optimal investment strategies under such a short- and long-term structure of risk. The serious challenge is not how to conceptually define such risks, but how to measure such risks.

In this section, we introduce a risk measure that is well-defined for both short- and long- term structure such that the risk measure is reduced to the short-term risk measure when one restricts to a show period of time horizon. The term structure of the risk measure is a natural analogue of the term structure of interest rate and the term structure of volatility as Engle (2011) studies.

Let $S_{t}$ be the value of a portfolio at time $\mathrm{t}$ and $s_{t}=\ln S_{t}$ be its $\log$ value. The loss is the difference $\left(S_{t+} T-S_{t}\right)$ between the period $T$ value and the current value of the portfolio. The term structure is with respect to the period $T$. Hence, the VaR at the level alpha and the time horizon $T$ per dollar invested is given by

$$
P_{t}\left[S_{t+T} / S_{t}-1<-V a R_{\alpha, T}\right]=\alpha
$$

The structure of risk requires the distribution of the underlying asset at time $t+T$ with a current information $\boldsymbol{F}_{t}$, the conditional probability with the loss is given by the predetermined quantile $\alpha$. In another word, for a given time horizon $T$ and the confidence level $\alpha$, the value at risk is the loss in market value over the time horizon $\mathrm{T}$ exceeded with probability 1- $\alpha$, Variations on VaR may represent forecasts of risk and returns.

Let $\operatorname{VaR}_{\alpha, T}^{0}$ be the $\alpha$ quantile of losses assuming expected continuously compounded returns are zero. So the expected losses from holding the asset can be estimated as

$$
P_{t}\left[s_{t+T}-E_{t} s_{t+T}<\ln \left(1-V a R_{\alpha, T}^{0}\right)\right]=\alpha
$$

Subtracting the term $s_{t}$ and moving the conditional expectation $E_{t} s_{t+T}$, we have

$$
\begin{aligned}
& P_{t}\left[s_{t+T}-s_{t}<E_{t} s_{t+T}-s_{t}+\ln \left(1-V a R_{\alpha, T}^{0}\right)\right]=\alpha \\
\text { Note that } P_{t}\left[S_{t+T} / S_{t}-1<-V a R_{\alpha, T}\right]= & \alpha \text { from }(1) . \text { Hence, } \\
& \ln \left(1-\operatorname{VaR}_{\alpha, T}\right)=E_{t} s_{t+T}-s_{t}+\ln \left(1-\operatorname{VaR}_{\alpha, T}^{0}\right)
\end{aligned}
$$

For any fixed period $T, E_{t} s_{t+T}=s_{t}$ if and only if $s_{t+T}$ is martingale if and only if $\operatorname{VaR}_{\alpha, T}=\operatorname{VaR_{\alpha ,T}^{0}}$. For any non-martingale $s_{t+T}$, we have the relation between the two measures of VaR approximately given by

$$
\operatorname{VaR}_{\alpha, T} \cong \operatorname{VaR}_{\alpha, T}^{0}-\mu T
$$

Where $\mu$ is the expected return (see also Engle (2011) for this) ${ }^{3}$. The bigger the estimate of the expected return $\mu$, the less risky the underlying asset appears confounding the separate estimation of risk and return. Since the expected returns are positive, the long horizon return is proportional to $T$. But the volatility is proportional to the square root of $T$, and the estimation of $\operatorname{VaR}_{\alpha, T}^{0}$ is proportional to the square root of $T$. Hence, by (3), $V a R_{\alpha, T}$ becomes negative for relatively large $T$ or the risk measure for the long-term $T$ becomes meaningless.

The Derivatives Policy Group proposes a standard quantile $\alpha=0.01$ and time horizon two weeks for over-the- counter derivatives broker-dealer reports to the Securities and Exchange Commission. This value at risk measures the 99\% confidence level of the probability distribution of changes in market value in the statistic sense. BIS (Bank for International Settleements) sets $\alpha=0.01$ and time horizon $T$ to 10 days for measuring the adequacy of bank capital, and $\mathrm{J}$. P. Morgan discloses the daily VaR at the confidence level $95 \%$. VaR measures only the aspect of market risk, and would

\footnotetext{
${ }^{3}$ Here one uses $\ln (1-x) \sim-x$ for sufficiently small $x$ and $E_{t} s_{t+T}-S_{t} \sim \mu T$ for non-convexity effects. So (3) follows from a simple algebra with these approximations.
} 
be relevant with some measure of cash ow at risk to better access capital adequacy ${ }^{4}$.

The risk measure given by $\operatorname{VaR}_{\alpha, T}$ is criticized (i) by theoretical point of view that it fails natural axioms of diversification, and (ii) by ignoring the size of the risk in the alpha tail. Value at Risk $\operatorname{VaR}_{\alpha, T}$ is usually computed over a day, a week or a few weeks by its approximation $V a R_{\alpha, T}^{0}-\mu T$. In real world, the approximated value $\operatorname{VaR}_{\alpha, T}^{0}-\mu T$ of $\operatorname{VaR}_{\alpha, T}$ cannot be adapted for long-term horizons. Instead of the previous approximation in (3), we define another extension $\operatorname{VaR}_{\alpha, T}$ of the risk measure $\operatorname{VaR}_{\alpha, T}$ to capture the long term risk measure in the sense that $\operatorname{VaR}_{\alpha, T}$ always measures the loss of the underlying asset ${ }^{5}$.

$$
P_{t}\left[s_{t+T}-E_{t} s_{t+T}-\mu T<\ln \left(1-\operatorname{VaR}_{\alpha, T}\right)\right]=\alpha
$$

Hence, the relation between the two measures of $\operatorname{VaR}_{\alpha, T}$ and $\operatorname{VaR}_{\alpha, T}^{0}$ is given by

$$
\ln \left(1-\operatorname{VaR}_{\alpha, T}\right)=\ln \left(1-\operatorname{VaR}_{\alpha, T}\right)-\mu T
$$

Therefore we obtain the extension of the risk measure

$$
\operatorname{VaR}_{\alpha, T}=e^{-\mu T} \operatorname{VaR}_{\alpha, T}^{0}+\left(1-e^{-\mu T}\right)
$$

The expression (6) shows that the risk measure is no longer negative for any large $T$, and for any $T>0$,

$$
\begin{gathered}
\operatorname{VaR}_{\alpha, T}>0, \quad \lim _{T \rightarrow+\infty} \operatorname{VaR}_{\alpha, T}=1 \\
\frac{\partial}{\partial T} \operatorname{VaR}_{\alpha, T}=\mu e^{-\mu T}\left(1-\operatorname{VaR}_{\alpha, T}^{0}\right)+e^{-\mu T} \cdot \frac{\partial}{\partial T} \operatorname{VaR}_{\alpha, T}^{0}
\end{gathered}
$$

The expression (6) is a nonlinear extension of the usual $V a R_{\alpha, T}$ since the linear expression of $\ln (1-x) \cong-x^{6}$ and replacing (5) by its linearization one gets (3).

The rate of the change with respect to the term structure $T$ is given by the sum of the positive rate of change term $\mu e^{-\mu T}\left(1-\operatorname{VaR}_{\alpha, T}^{0}\right)$ and the discounted version of the rate of change for the logarithmic mean zero risk measure from (6). It is interesting to see that the nonlinear extension $\operatorname{VaR}_{\alpha, T}$ eventually increases to its maximum value 1 at the infinity term structure.

From the term structure point of view and (2), we have

$$
\left(1-\operatorname{VaR}_{\alpha, T}\right) /\left(1-\operatorname{VaR}_{\alpha, T}\right)=e^{E_{t} s_{t+T}-s_{t}+\mu T}
$$

By (7), we have $V a R_{\alpha, T}=V a R_{\alpha, T}$ if and only if $E_{t} s_{t+T}=s_{t}-\mu T ; \quad V a R_{\alpha, T}>V a R_{\alpha, T}$ if and only if $E_{t} s_{t+T}-s_{t}+\mu T<0$; and $\operatorname{VaR}_{\alpha, T}<\operatorname{VaR} R_{\alpha, T}$ if and only if $E_{t} s_{t+T^{-}} s_{t}+\mu T>0$. This $\operatorname{VaR} R_{\alpha, T}$ relaxes the martingale condition $E_{t} s_{t+T}=s_{t}$ to have a better approximation of $\operatorname{VaR}_{\alpha, T}$ than $\operatorname{VaR}_{\alpha, T}^{0}$. We obtain

$$
V a R_{\alpha, T}=\left(1-e^{-E_{t} s_{t+T}+s_{t}-\mu T}\right)+V a R_{\alpha, T} e^{-E_{t} s_{t+T}+s_{t}-\mu T}
$$

where $\operatorname{VaR}_{\alpha, T}>\operatorname{VaR} R_{\alpha, T}^{0}$ is a better approximation of $\operatorname{VaR}_{\alpha, T}$ for large $T$.

\footnotetext{
${ }^{4}$ The true Value at Risk is much bigger tan the computed VaR if there are political risk, liquidity risk and regulatory risks associated to the underlying asset that are not built into the VaR.

${ }^{5}$ Here one uses $\ln (1-x) \sim-x$ for sufficiently small $x$ and $E_{t} s_{t+T}-S_{t} \sim \mu T$ for non-convexity effects. So (3) follows from a simple algebra with these approximations.

The definition of $V a R_{\alpha, T}$ in (4) has an important sign issue we would like to point out here. For a small $T$ in the linear approximation, we have $\operatorname{VaR}_{\alpha, T}=\operatorname{VaR}_{\alpha, T}^{0}-\mu T>\operatorname{VaR}_{\alpha, T}^{0}$. If one directly replaces $s_{t}$ by $E_{t} s_{t+T^{-}} \mu T$ to define the new Value at Risk

$P_{t}\left[s_{t+T}-\left(E_{t} s_{t+T}-\mu T\right)<\ln \left(1-\operatorname{VaR}_{\alpha, T}^{\prime}\right)\right]=\alpha$, then the relation between $\operatorname{VaR}_{\alpha, T}^{\prime}$ and $\operatorname{VaR}_{\alpha, T}^{0}$ is given by

$$
\left(1-\operatorname{VaR}_{\alpha, T}^{\prime}\right) /\left(1-\operatorname{VaR}_{\alpha, T}^{0}\right)=e^{\mu T}>1
$$

by a straightforward calculation. Therefore this definition $\operatorname{VaR}_{\alpha, T}^{\prime}$ of the Value at Risk leads to $\operatorname{VaR}_{\alpha, T}^{\prime}<\operatorname{VaR} R_{\alpha, T}^{0}$ for all $T>0$ and further far away from $\operatorname{VaR}_{\alpha, T}$. The adjustment of the new measurement given in (4) serves to resolve this issue and to resolve the negative problem for a long time horizon as well.

${ }^{6}$ It is clear that $-\ln (1-x)=x+x^{2} / 2+x^{3} / 3+\cdots+x^{n} / n+\cdots$ holds for $|x|<1$ and the linearization of $\ln (1-x)$ at $x=0$ is simply $-x$.
} 
To summarize the relations among three different VaR's, we make the following characteristics.

(1) $\operatorname{VaR}_{\alpha, T}^{0}=\left(1-e^{-E_{t} s_{t+T}+s_{t}}\right)+\operatorname{VaR}_{\alpha, T} e^{-E_{t} s_{t+T}+s_{t}}$ and $E_{t} s_{t+T}-S_{t}=O(T)$.

$1-\operatorname{VaR}_{\alpha, T}^{0}=\left(1-\operatorname{VaR}_{\alpha, T}\right) e^{-E_{t} s_{t+T}+s_{t}}$, where $\operatorname{VaR}_{\alpha, T}^{0}=\operatorname{VaR}_{\alpha, T}$ if and only if $E_{t} s_{t+T}=s_{t}$ for any $T$.

(2) $V a R_{\alpha, T}=\left(1-e^{-E_{t} s_{t+T}+s_{t}-\mu T}\right)+\operatorname{VaR}_{\alpha, T} e^{-E_{t} s_{t+T}+s_{t}-\mu T}>0$ and

$1-\operatorname{VaR}_{\alpha, T}=\left(1-\operatorname{VaR}_{\alpha, T}\right) e^{-E_{t} s_{t+T}+s_{t}-\mu T}$, where $\operatorname{VaR} \alpha, T=\operatorname{VaR} R_{\alpha, T}$ if and only if $E_{t} s_{t+T}=s_{t}-\mu T$.

In general, these three VaR's are indeed different. The earlier $\operatorname{VaR}_{\alpha, T}^{0}$ can be thought of a linear approximation of $V a R_{\alpha, T}$ in terms of $T$, and our extension $\operatorname{VaR} R_{\alpha, T}$ can be thought of a better approximation of $\operatorname{VaR} R_{\alpha, T}$ above $\operatorname{VaR}_{\alpha, T}^{0}$ for all $T$. But most calculations of $\operatorname{VaR}_{\alpha, T}^{0}$ take further simplification from $\ln (1-x)=-x$ to $\operatorname{VaR}_{\alpha, T}^{0}=\operatorname{VaR}_{\alpha, T}-\left(E_{t} s_{t+T}-s_{t}\right)$. Regardless of simplification, our extension $\operatorname{VaR} \alpha, T>0$ gives better understanding of the true VaR for any $\mathrm{T}$ than $\operatorname{VaR}_{\alpha, T}^{0}$ does.

Now we use the nonlinear extension $\operatorname{VaR}_{\alpha, T}$ of the risk measure to include the size of the risk and define the expected loss should $\operatorname{VaR}_{\alpha, T}$ be exceeded. Therefore

$$
E S_{\alpha, T}=-E_{t}\left[S_{t+T} / S_{t}-1 \mid S_{t+T} / S_{t}-1<-\operatorname{VaR}_{\alpha, T}\right]
$$

For the mean logarithmic return zero, we have the expected loss is given by

$$
E S_{\alpha, T}^{0}=-E_{t}\left[S_{t+T} / S_{t}-1 \mid S_{t+T} / S_{t}-1<-V a R_{\alpha, T}^{0}\right] .
$$

By using extreme value theory, the Pareto distribution is given by

$$
F(x)=1-\left(x / x_{0}\right)^{-\lambda}, 0<x_{0} \leqslant x,
$$

where the only parameter $\lambda$ is required to estimate the probability. The tails of the distribution get narrower as the parameter increases. The Pareto density function is given by $F^{\prime}(x)=f(x)=\lambda\left(x / x_{0}\right)^{-\lambda-1}$ for $0<x_{0}<x$. By setting $x_{0}=V a R_{\alpha, T}^{0}$ and $x_{t}=1-s_{t+T} / s_{t}$, one has

$$
E S_{\alpha, T}^{0}=-E_{t}\left[x_{t} \mid x_{t}>x_{0}\right]=\operatorname{VaR}_{\alpha, T}^{0} \cdot \lambda /(\lambda-1),
$$

for $\lambda>1$. The common approach to the parameter $\lambda$ is to use the Hill estimator. McNeil, mbrechts, and Frey (2005) define a threshold $x_{0}$ so that all data exceeding this $x_{0}$ follows the Pareto distribution. This will be used to form a maximum likelyhood estimator of the unknown tail parameter. Hence, the Hill estimator is

$$
z_{t}=\frac{1-S_{t+T} / S_{t}}{\operatorname{VaR}_{\alpha, T}^{0}}, \hat{\lambda}=\sum_{t \in \text { Exceed }} 1 / \sum_{t \in \text { Exceed }} \ln \left(z_{t}\right)
$$

Resnick and Starica (1995) and references therein show that Hill's estimators in (9) is consistent for the tail index of the marginal distribution in a time series context provided the marginal distribution is independent of the time.

Assume that the long term tail beyond $\operatorname{VaR}_{\alpha, T}$ follows the Pareto distribution, and with the Hill's estimator $\hat{\lambda}$, the lower tail by focusing on losses $1-S_{t+T} / S_{t}$,

$$
E S_{\alpha, T}=\operatorname{VaR}_{\alpha, T} \cdot \hat{\lambda} /(\hat{\lambda}-1)
$$

\section{Coherent Risk Measurement from $\operatorname{VaR}$}

Artzner et al (1997, 1999) introduce a constructive evaluation for the capital requirement with four basic desired properties, since the managing risk by VaR may fail to stimulate diversification and VaR does not satisfy the sub-additive property (see Embrechts (2000) for review on this criticism). Acerbi et al (2002) define the expected shortfall which is both coherent and easy to compute and estimate. The expected shortfall as a coherence risk measure is easier to compute than the tail conditional expectation and the worst conditional expectation defined by Artzner et al (1999).

For our extended $V a R$, we define the corresponding tail conditional expectance, $\alpha$-tail mean and the expected shortfall to formulate both coherent and easy risk measure. For the fixed quantile $\alpha$, we define the tail conditional expectation at a level $\alpha$ is the measure of risk given by

$$
T C E_{T}^{\alpha}=-E_{t}\left[X_{t, T} \mid X_{t, T} \leq-V_{a} R_{\alpha, T}\right]
$$

where $X_{t, T}=S_{t+T} / S_{t}-1$ in our case. Delbaen (2002) shows that $T C E^{\alpha}$ in general is not subadditive in Theorem 6.10. Hence, 
the worst conditional expectation is defined in Artzner et al (1999) Definition 5.2. The worst conditional expectation is sub-additive, but the smallest coherent risk measure dominating $\operatorname{VaR}_{\alpha, T}$. It is extremely difficult to compute. We follow Acerbi et al (2002) to extend the corresponding tail mean and expected shortfall for the $\operatorname{VaR} \alpha, T$. The extended $\alpha$-tail mean $\operatorname{VaR}_{\alpha, T}$ is defined by

$$
T M_{\alpha, T}\left(X_{t, T}\right)=\frac{1}{\alpha}\left(E_{t}\left[X_{t, T} \mid X_{t, T} \leq-\operatorname{VaR}_{\alpha, T}\right]+\operatorname{VaR}_{\alpha, T}\left(P_{t}\left[X_{t, T} \leq-\operatorname{VaR} R_{\alpha, T}\right]-\alpha\right)\right)
$$

The extended expected shortfall is defined by

$$
E S_{\alpha, T}\left(X_{t, T}\right)=-T M_{\alpha, T}\left(X_{t, T}\right)
$$

Acerbi et al (2002) show that $E S_{\alpha}$ is continuous with respect to $\alpha$, and is sub-additive. By the same proof, we have the following proposition.

Proposition 3.1. For a fixed quantile $\alpha \in(0,1)$ and a fixed long term $\mathrm{T}$, consider a set $V_{t, T}$ of real-valued random variables on a probability space $\left(\Omega_{T}, \mathbf{A}_{t}, P_{t}\right)$ for the present time $t$ such that $E_{t}\left[\max \left\{-X_{t, T}, 0\right\}\right]<\infty 1$ for all $X_{t, T} \in V_{t, T}$. Then $\rho_{\alpha, T}: V_{t, T} \rightarrow \mathbf{R}$ with $\rho_{\alpha, T}\left(X_{t, T}\right)=E S_{\alpha, T}\left(X_{t, T}\right)$ for $X_{t, T} \in V_{t, T}$ is a coherent risk measure in the sense of Definition 2.4 of Artzner et al (1999) and Definition 2.1 in Delbaen (2002): i.e., the risk measure $\rho_{\alpha, T}$ satisfies the monotonicity, sub-additivity, positive homogeneity and translation invariance ${ }^{7}$.

For the quantile $\alpha=0.01$, we can evaluate the extended tail condition expectation $T C E_{T}^{\alpha}$ at the level 0.01 , the extended $\alpha$-tail mean $T M_{\alpha, T}$ and the extended expected shortfall $E S_{\alpha, T}\left(X_{t, T}\right)$ for S\&P 500 from Jan. 2, 1990 to Dec. 31, 2012. We also compute the corresponding extended risk measures for both the Hong Kong Hang Seng index (HSI) and the Chinese Hushen 300 index (SHSZ 300) in the following Table 1-Table 6.

\section{Estimating $V a R$ for both Short- and Long-Terms}

In this section we compare both $\operatorname{VaR}$ and $\mathrm{VaR}$ for S\&P 500 for both short- and longterms. Using the empirical data, we test the behavior of $V a R$. We analyze the short- and long-term skewness and kurtosis for S\&P 500 .

\subsection{Comparing VaR with VaR for S\&P 500}

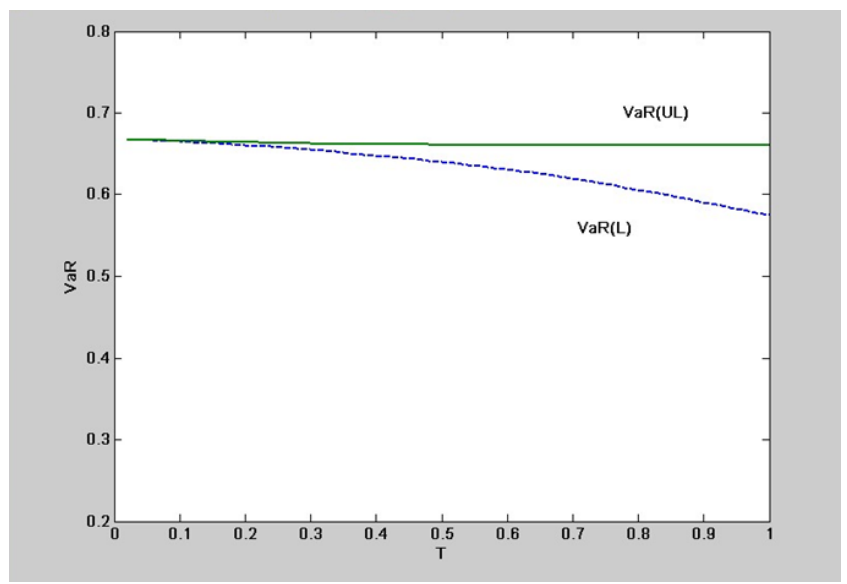

Figure 1. short-term VaR of S\&P 500

In order to compare the risk measure $V a R$ with the risk measure VaR used in Engle (2011), we use data on S\&P 500 from Jan. 2, 1990 to Dec. 31, 2012. The step we choose is a week $5 / 251$ as 0.02 . By using IBM SPSS Statistics, we test that the sequence $\left\{s_{t+0.02}-E_{t} s_{t+0.02}\right\}$ follows the normal distribution with mean and standard deviation. Hence, $\operatorname{VaR}^{0}$ and $\mu$ can be determined correspondingly with the quantile $\alpha=0.01$.

Figure 1 shows the graph of both $\operatorname{VaR}$ (denoted by $\operatorname{VaR}(U L)$ ) and $\operatorname{VaR}($ denoted by $\operatorname{VaR}(L)$ ) curves for one year $T=1$

\footnotetext{
${ }^{7}$ The proof of this proposition follows exactly the same proof of Proposition 3.1 of Acerbi and Tasche (2002) in the Appendix A.
} 
case). Both two curves decrease until $T=0.57$, where $\operatorname{VaR}(U L)$ and VaR are almost identical near the period [0,0.3] (up to 15 weeks, $\operatorname{VaR}$ can be estimated as VaR). But the variation starts to change during the period [0.57, 0.67] where $\operatorname{VaR}(L)$ evaluated by Engle (2011) decreases faster than our risk measure $\operatorname{VaR}$, and at $T=0.9 \operatorname{VaR}(U L)$ starts to increase slowly.
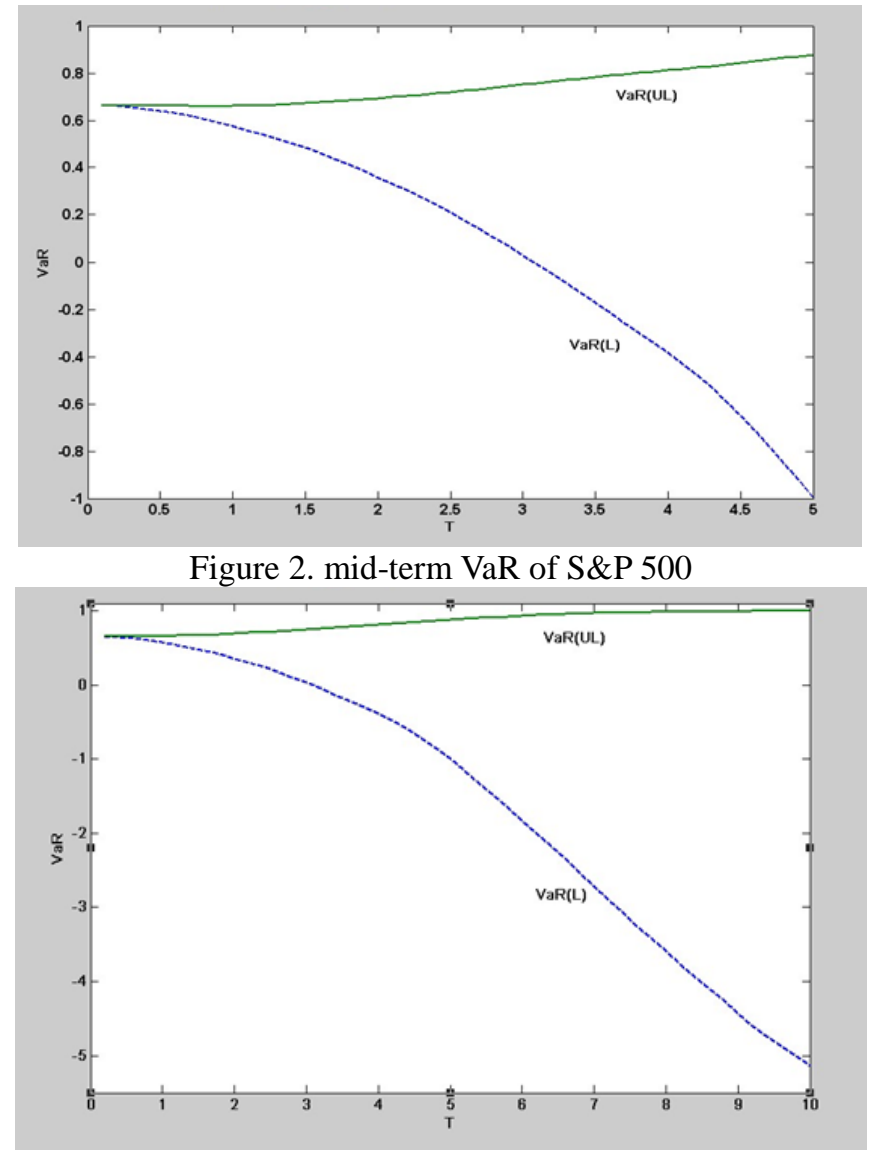

Figure 3. long-term VaR of S\&P 500

Figure 2 shows the risk measurements over the 5 years period $T=5$ with a step 0.1 which is about one month with the quantile $\alpha=0.01$. The decreasing behavior of the curve $\operatorname{VaR}(L)$ continues and reaches to negative values after $T=3.1$. This is typically predicted by Engle (2011) that $\operatorname{VaR}(L)$ does not matter for short horizon whether expected returns are considered or not but for longer horizons $\operatorname{VaR}(L)$ becomes negative. For S\&P 500, the risk measure goes to negative after 13 months. On the other hand, our risk measure $\operatorname{VaR}$ (denoted by $\operatorname{VaR}(U L)$ ) decreases gradually first and tips up at $T=0.9$. During the period of 5 years, the curve $\operatorname{VaR}(L)$ stays in positive range and gives a meaningful extension of the long-term risk measure. This is further verified for taking an even longer period $T=10$ as in Figure 3.

\subsection{Long-term skewness and kurtosis for $S \& P 500$}

Engle (2004) in his Noble lecture argues that asymmetric volatility models generate multi-period returns with negative skewness even if the innovations are symmetric. Engle (2004) further states that the kurtosis of the nineties is substantial at 6.8, while for the full sample it is a dramatic 41. This is strong evidence that extremes are more substantial than would be expected from a normal random variable ${ }^{8}$. The negative returns predict higher volatilities than positive returns, the high volatility after negative returns means that the possible market declines are more extreme than the possible market increases (see Berd, Engle and Voronov (2007) and Engle (2011)).

The skewness is defined in terms of long horizon continuously compounded as

\footnotetext{
${ }^{8}$ That the kurtosis measures the magnitude of the extremes is the most interesting feature. If returns are normally distributed, then the kurtosis should be three.
} 


$$
s k_{t}(T)=\frac{E_{t}\left(s_{t+T}-s_{t}-\mu\right)^{3}}{\left[E_{t}\left(s_{t+T}-s_{t}-\mu\right)^{2}\right]^{3 / 2}}, \mu_{t}(T)=E_{t}\left(s_{t+T}-s_{t}\right)
$$

With log return, $s k_{t}(T)$ measures focuses on asymmetric of the distribution and provides a systematic deviation from symmetry and negative skewness means that large declines are more likely than similar size increases. If one holds long positions, then negative skewness is a source of concern for $\mathrm{VaR}$, as the large negative returns are more likely than large positive returns in the sense of skewness.

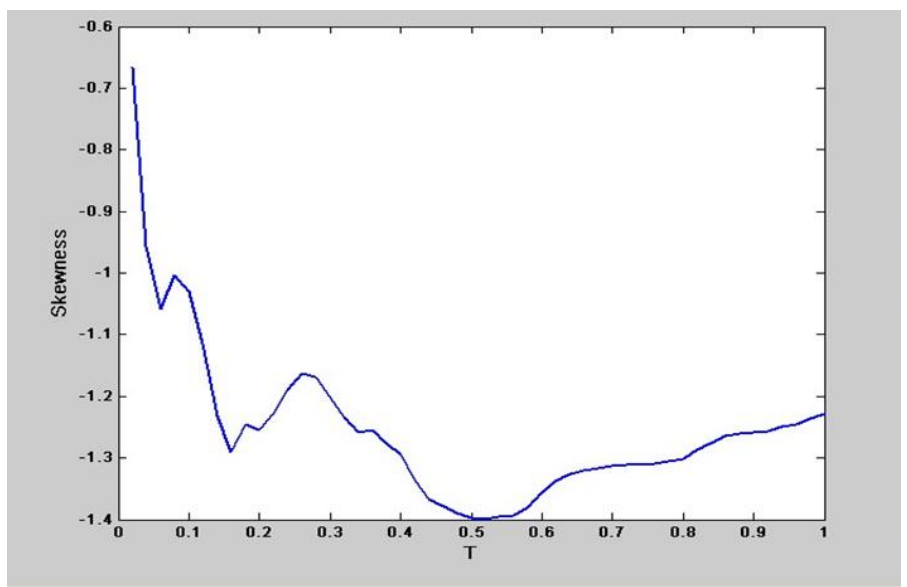

Figure 4. short-term skewness of S\&P 500

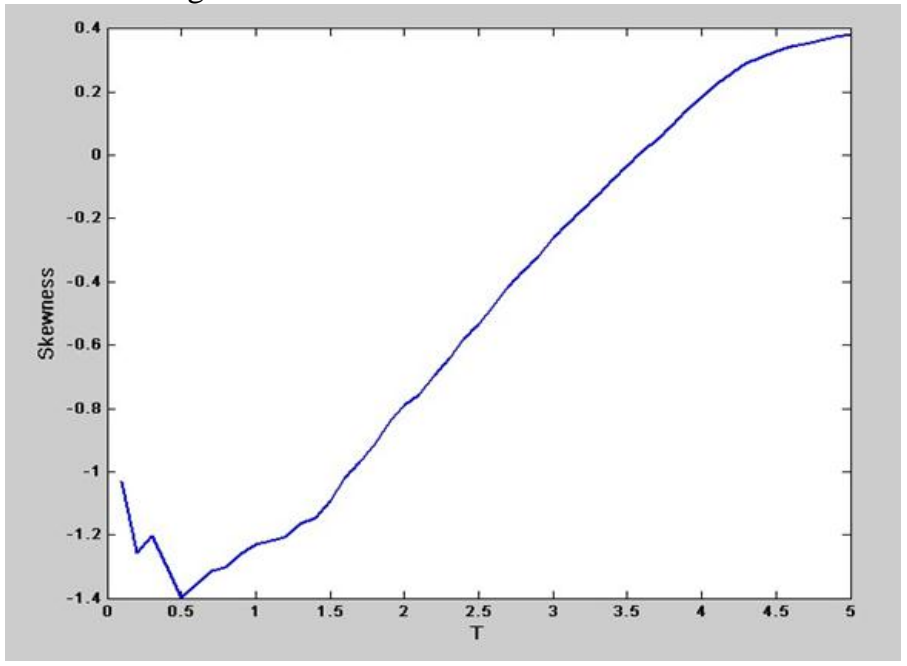

Figure 5. mid-term skewness of S\&P 500

Figure 4 for the one year horizon shows that the skewness for $\mathrm{T}=0.04,0.06 ; \cdots, 1$ are all negative and continuously decreasing until $T=0.6$ about 30 weeks. The skewness starts to increase after $T=0.6$. For the first 1 to 100 days, our Figure 4 is same as Figure 6 of Engle (2011). But we look for even longer horizon so that the skewness measures are negative, and are not increasingly negative for longer horizon. This improves the Engle (2011)'s findings ${ }^{9}$. Engle (2011) already realizes this fact that the data appears to be more negatively skewed for short horizons and less negatively skewed for long horizons than the models, and develops an econometric test to determine whether the long-term skewness implied by a set of parameter estimates is significantly different from that in the data in section 4 . The Monte Carlo critical values are computed, and the models cannot be reject for misspecification of long-term skewness. All those different models (GARCH, TARCH, EGARCH, APARCH, NGARCH, ASQGARCH) appear to generate long-term skewness that is consistent with the S\&P data. As we extend the longer horizon from one year to five year, Figure 5 shows the skewness continuously increases after $T=0.6$ and changes from negative to positive around $T=3.55$. When one looks for even longer horizon $T=10$ years, the skewness curve in Figure 6 shows that the skewness starts negative and increasingly negative until $T=3.55$, then increases from negative to positive until $T=6.6$, and finally decreases after $T=6.6$. This reects the high volatility of the possible market declines and increases during this 10 years.

\footnotetext{
${ }^{9}$ Engle (2011) on page 447 states that all the skewness measures are negative and are increasingly negative for longer horizons. For the same S\&P 500, we find that the skewness are not increasingly negative for longer horizon in Figure 4. The U-shape indicates the turning point for the possible market increasing.
} 


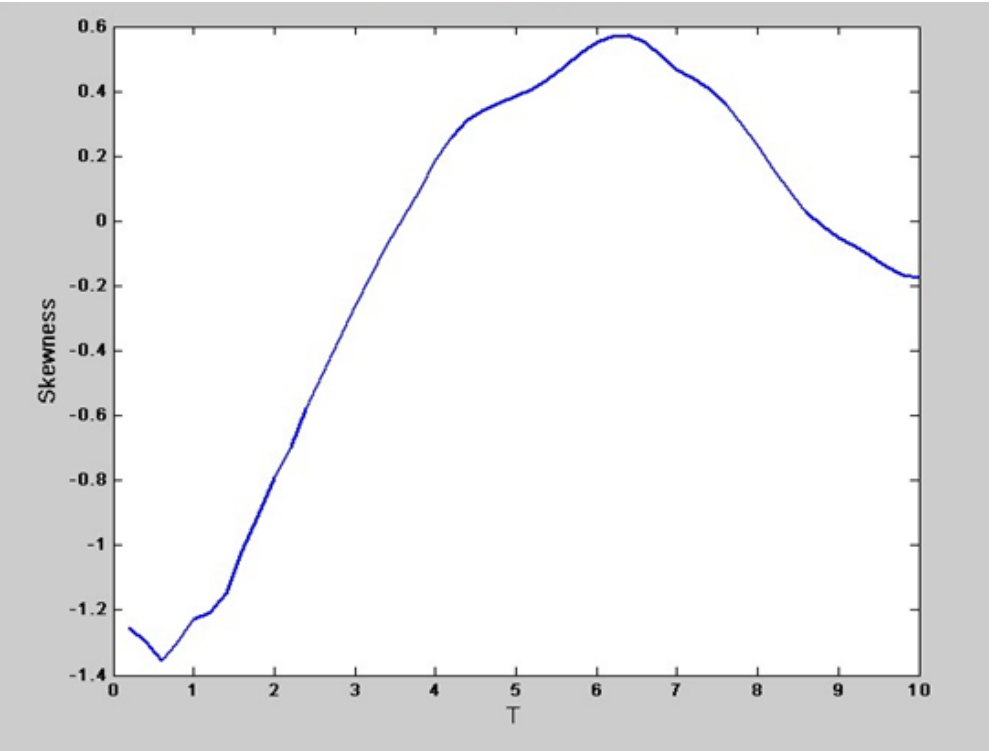

Figure 6. long-term skewness of S\&P 500

The kurtosis is de_ned by the following,

$$
k u_{t}(T)=\frac{E_{t}\left(s_{t+T}-s_{t}-\mu\right)^{4}}{\left[E_{t}\left(s_{t+T}-s_{t}-\mu\right)^{2}\right]^{2}}-3, \mu_{t}(T)=E_{t}\left(s_{t+T}-s_{t}\right)
$$

The kurtosis measures the tail-fatness. Duffie and Pan (1997) show that the S\&P 500 daily returns for 1986 to 1996 have an extremely high sample kurtosis of 111, in large measure due to the exceptional returns associated with the market crash of October 1987. At the quantile 0.01, the S\&P 500 historical returns for 1986 to 1996 is about 2.49 standard deviations from the mean. Fat-tails measured as the kurtosis, the behavior and estimates of volatilities are key issues to understand the price risk. The kurtosis estimates are highly sensitive to extremely large return.

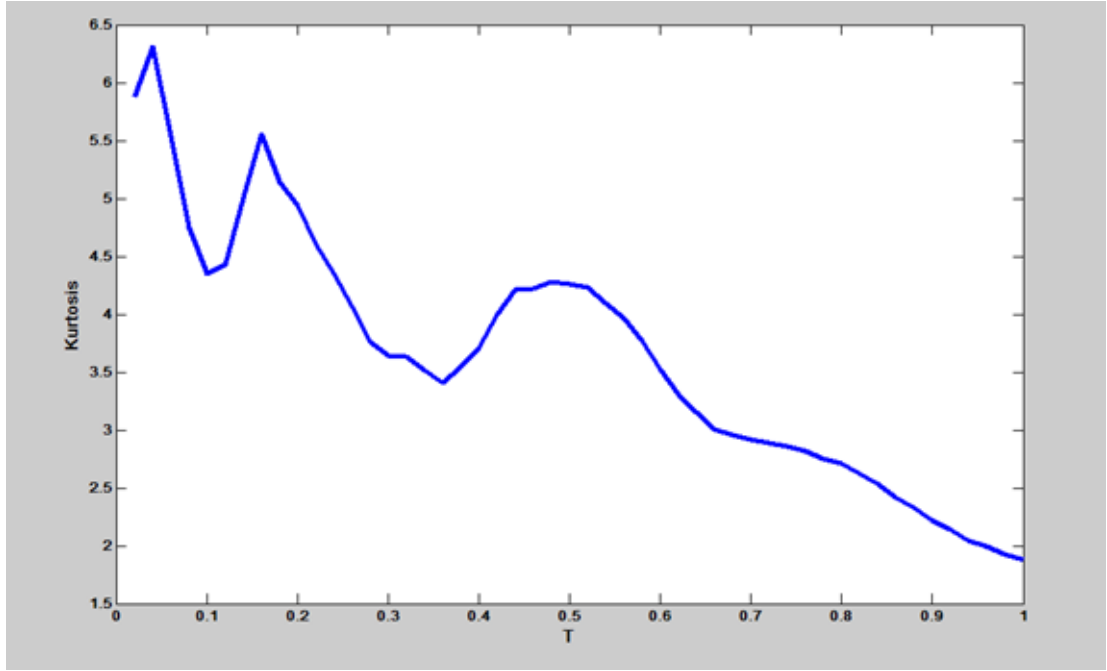

Figure 7. short-term kurtosis of S\&P 500 


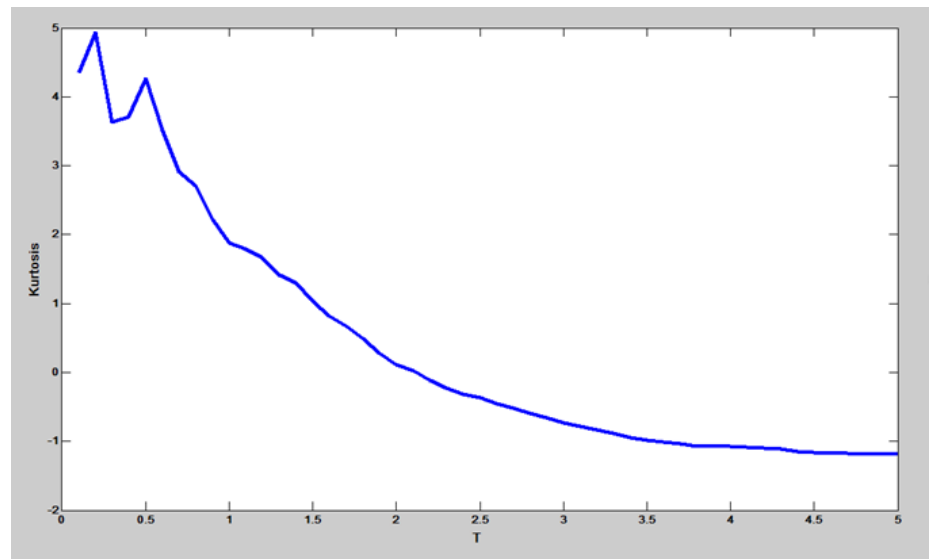

Figure 8. mid-term kurtosis of S\&P 500

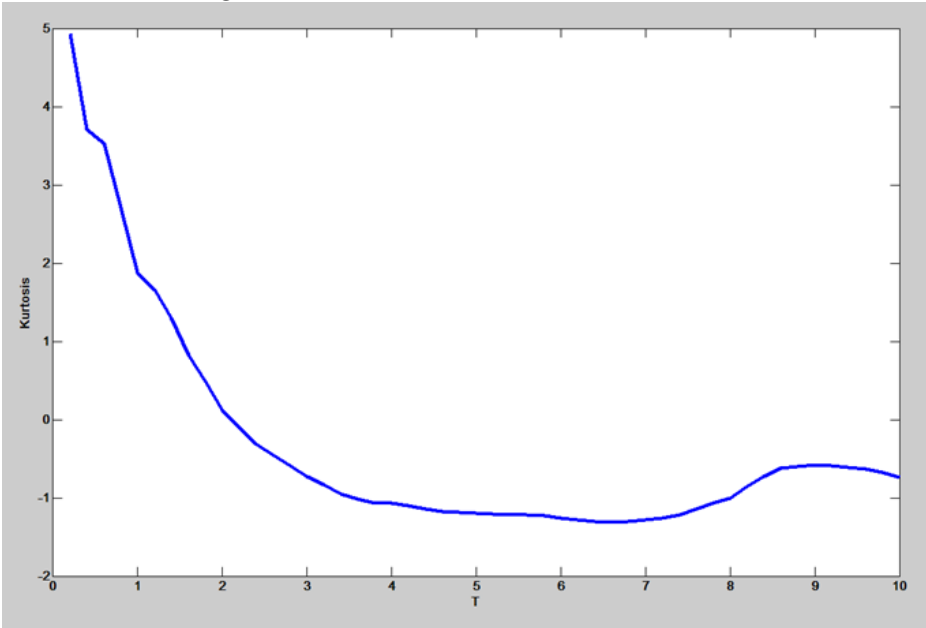

Figure 9. long-term kurtosis of S\&P 500

Figure 7 shows that for one year horizon the kurtosis with 0.02 steps is zigzagged and gradually decreased in this period. Figure 8 for five year horizon indicates the decreasing behavior continues to reach to zero around $T=2.15$ and tends to negative value (below the standard kurtosis 3). See also Figure 9 for the longer time kurtosis.

\section{Long-term structure of $\mathrm{VaR}$, skewness and kurtosis for HSI index}

In this section, we adopt the method developed in previous section to empirically test the Hong Kong Hang Seng index (HSI) and the chinese Hushen 300 index (SHSZ 300) on the VaR, skewness and kurtosis.

\subsection{Long term VaR for HSI}

We use the HSI data from Jan. 2, 1990 to Dec. 31, 2012 to analyze the time horizon from one year, five year and ten years separately. For the reason that the average trading days of HSI is 249 every year, the short-term step we choose is a week $5 / 249$ as 0.02 , the mid-term step we choose is a month $25 / 249$ as 0.1 , the long-term step we choose is two months $50 / 249$ as 0.2 .

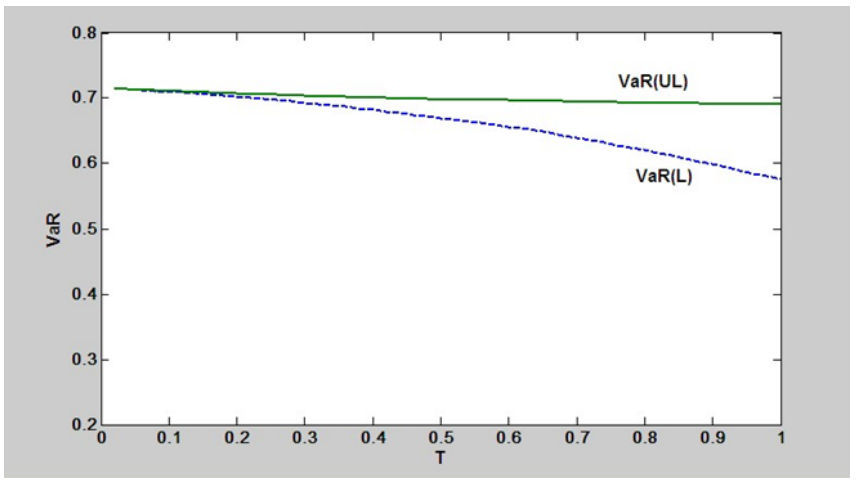

Figure 10. short-term VaR of HSI 


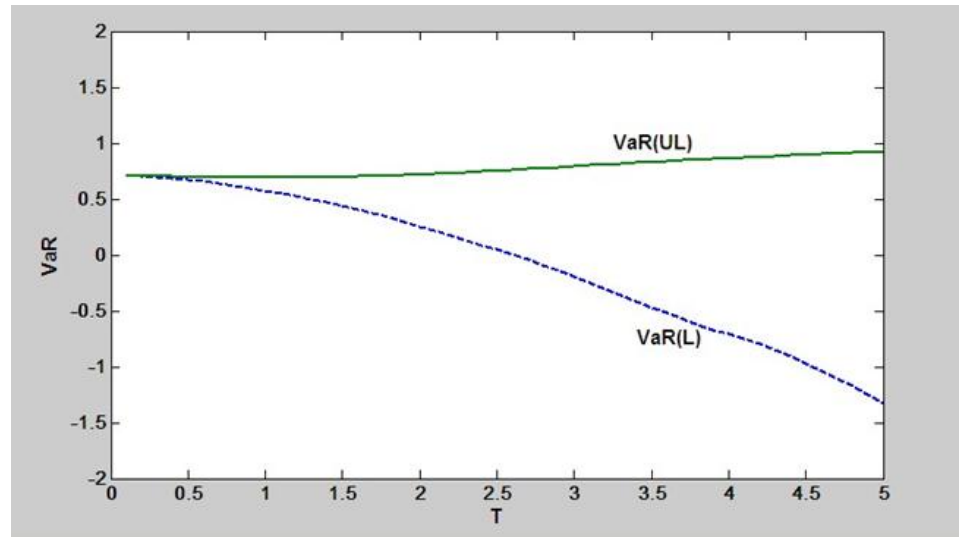

Figure 11. mid-term VaR of HSI

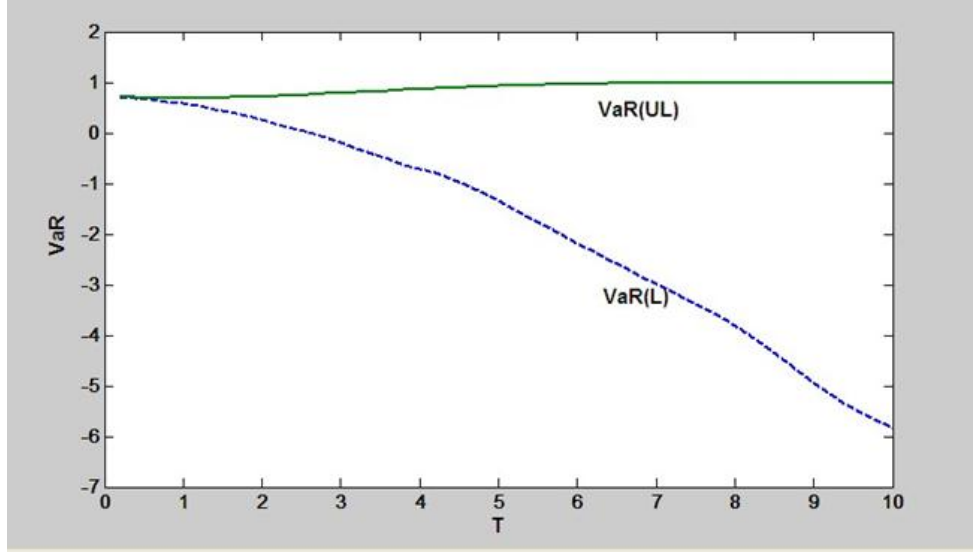

Figure 12. long-term VaR of HSI

Figure 10 shows that for one year horizon the $\operatorname{VaR}(U L)$ and $\operatorname{VaR}(L)$ are almost identical near the very short term horizon and then $\operatorname{VaR}(L)$ evaluated by Engle (2011) decreases faster than our risk measure $\operatorname{VaR}$, and at $T=0.9 \operatorname{VaR}(U L)$ starts to increase slowly.

Figure 11 shows the same property that $\operatorname{VaR}(L)$ starts to reach zero around $T=2.7$ for HSI as Figure 2 for S\&P 500. The curve $\operatorname{VaR}(L)$ continuously decreases. The curve $\operatorname{VaR}$ decreases slower than the curve $\operatorname{VaR}(L)$ and starts to increase at $T=1.2$ for HSI to illustrate the similar U-shape for the Hong Kong Hang Seng index (HSI).

\subsection{Long-term skewness and kurtosis for HSI}

Figure 13 shows that the short term skewness is also negative between -0.5 and -0.75 . Unlike the increasing negative skewness of S\&P 500 for the one year horizon which is more negative than HSI. The systematic deviation from symmetry of HSI is smaller than the one of S\&P 500, and HSI negative skewness means that large declines are more likely than similar size increases. But compared with S\&P 500, HSI decline is smaller size-wise.

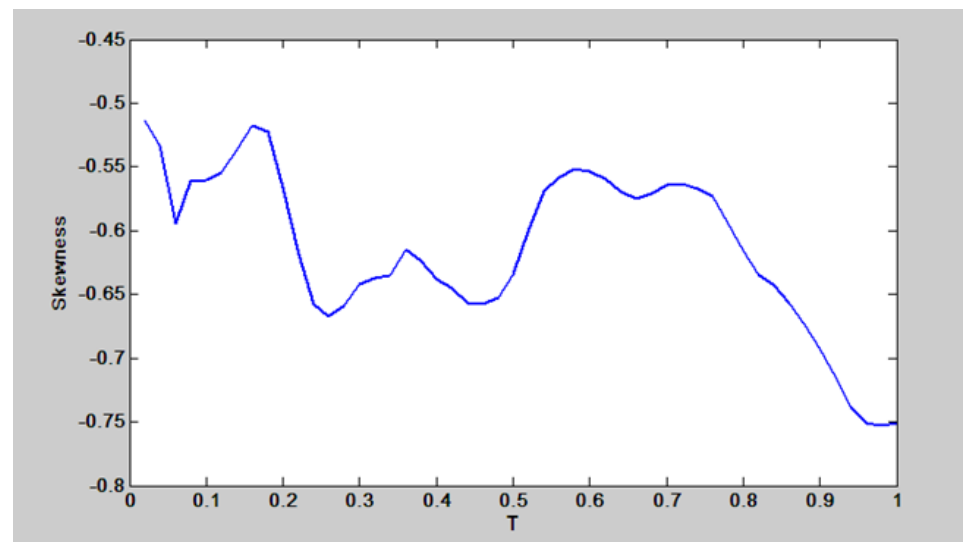

Figure 13. short-term skewness of HSI 


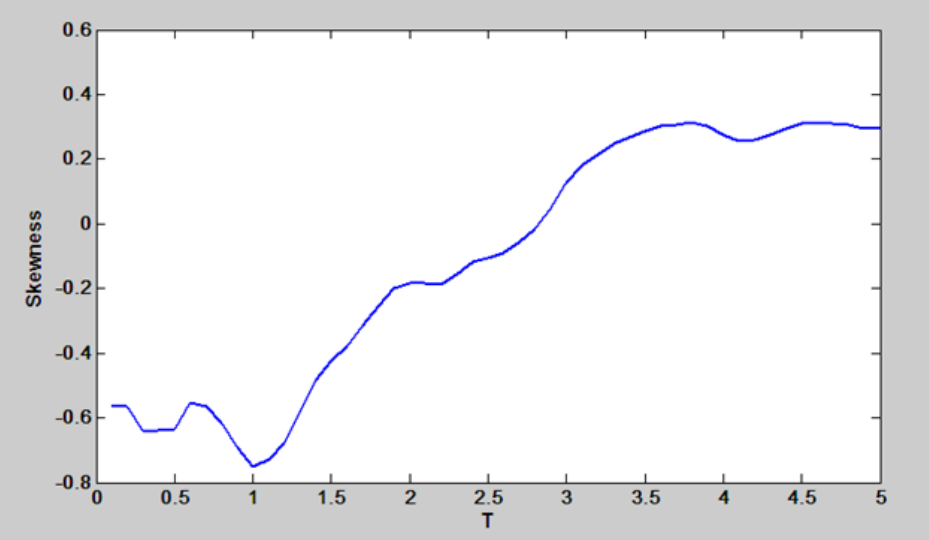

Figure 14. mid-term skewness of HSI

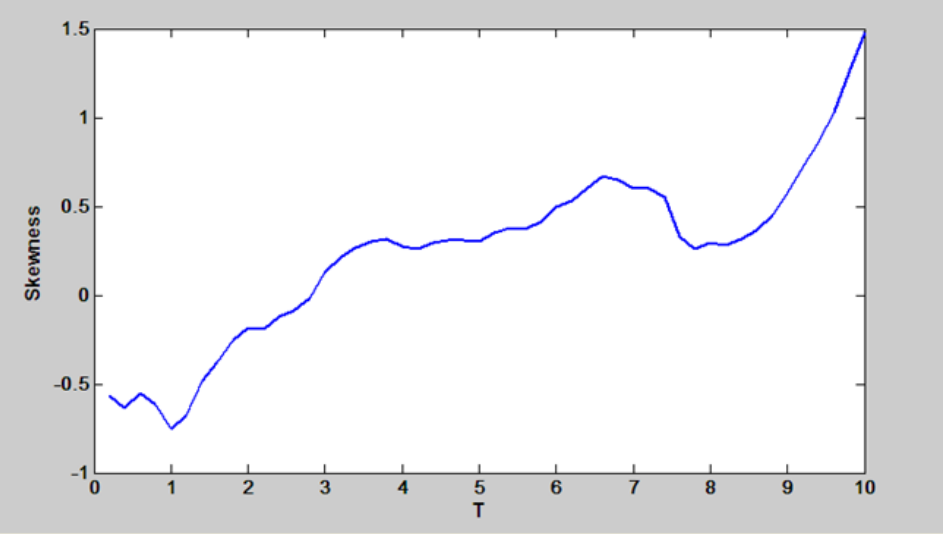

Figure 15. long-term skewness of HSI

Figure 14 for the five year horizon shows that the skewness starts to be positive after $T=2.85$. See also Figure 15 for the ten year horizon case.

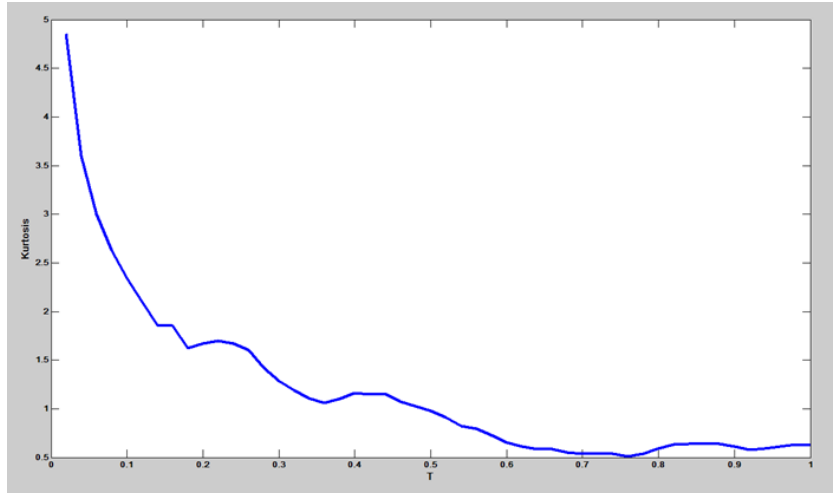

Figure 16. short-term kurtosis of HSI

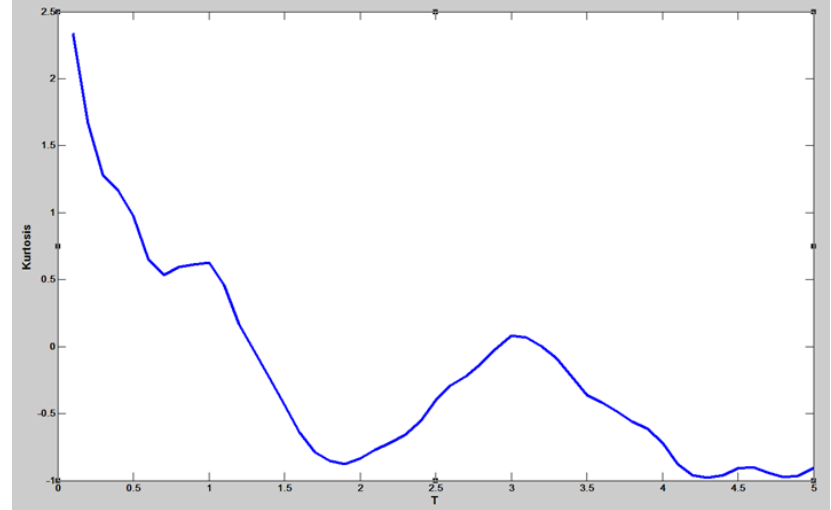

Figure 17. mid-term kurtosis of HSI 


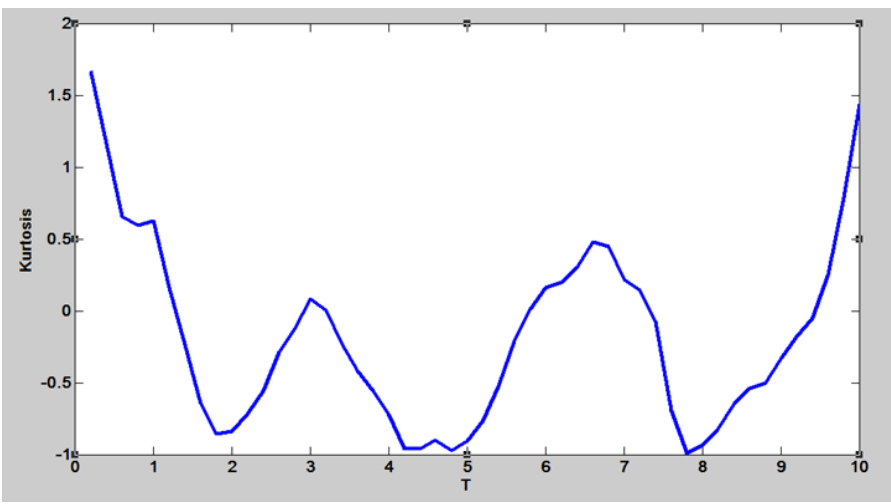

Figure 18. long-term kurtosis of HSI

Figure 16 shows that for one year horizon the kurtosis decreases for HSI and close to the kurtosis of a normally distributed shock. By this measure, HSI returns are not particularly fat-tailed at the level 0.01 . The quantile $\alpha=0.01$ for HSI historical returns for one year horizon approximately has 3.5 kurtosis.

For the five year horizon the kurtosis of HSI is near the kurtosis of a normally distributed shock. The Hong Kong HSI shows that return shocks have no fatter-than-normal tails, measured by kurtosis at the confidence level $\alpha=0.01$. Similarly the ten year horizon kurtosis is presented in Figure 18.

\subsection{Long term VaR for SHSZ 300}

We use the SHSZ 300 data from Jan. 4, 2002 to Dec. 31, 2012 to analyze the time horizon from one year, five year and ten years separately. For the reason that the average trading days of HSI is 242 every year, the short-term step we choose is a week $5 / 242$ as 0.02 , the mid-term step we choose is a month $25 / 242$ as 0.1 , the long-term step we choose is two months $50 / 242$ as 0.2 .

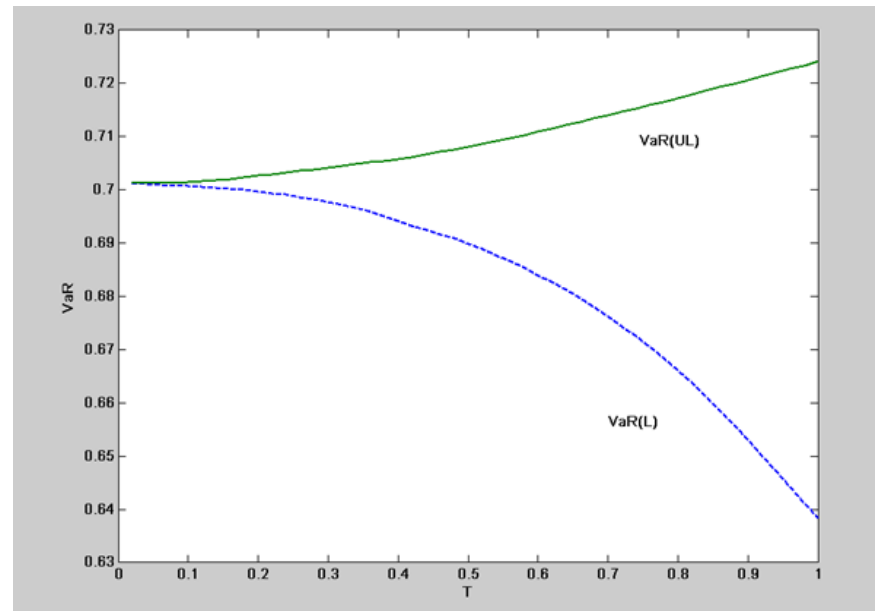

Figure 19. short-term VaR of SHSZ 300

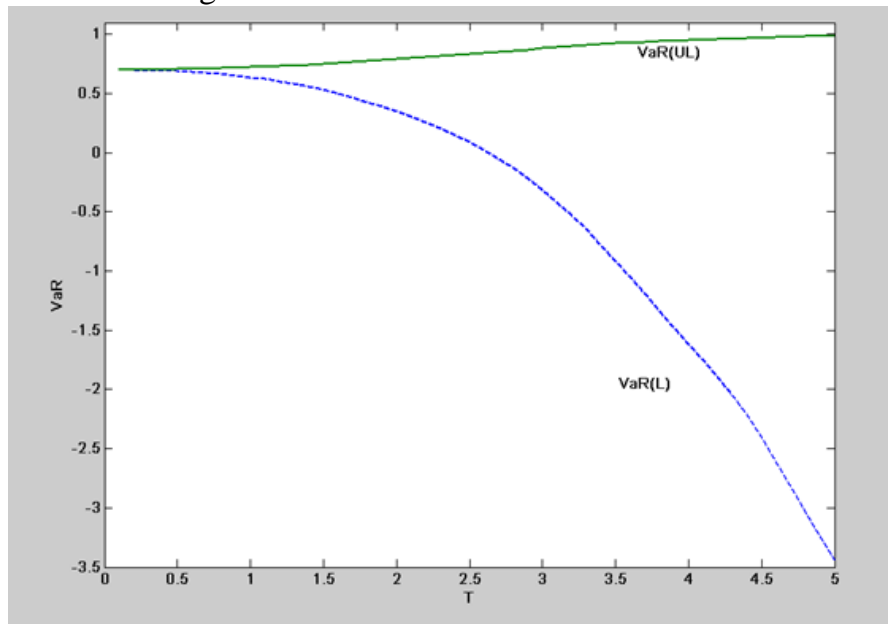

Figure 20. mid-term VaR of SHSZ 300 


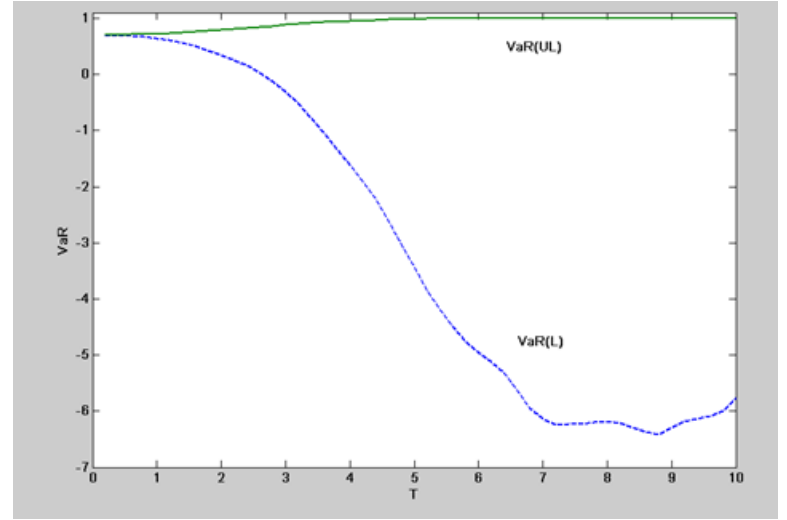

Figure 21. long-term VaR of SHSZ 300

Figure 19 compares both curves of $\operatorname{VaR}$ and $\mathrm{VaR}$ for one year horizon. The curve $\operatorname{VaR}(\mathrm{L})$ decreases and $\operatorname{VaR}(U L)$ increases for SHSZ 300. For the five year horizon, we see the VaR continues to decrease to negative value after $T=2.65$, where the curve $\operatorname{VaR}(U L)$ stays in the positive range and starts to approach to 1.

\subsection{Long-term skewness and kurtosis for SHSZ 300}

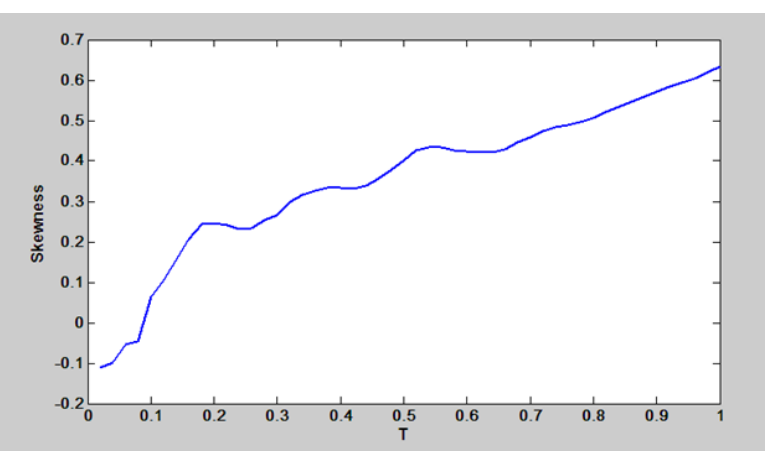

Figure 22. short-term skewness of SHSZ 300

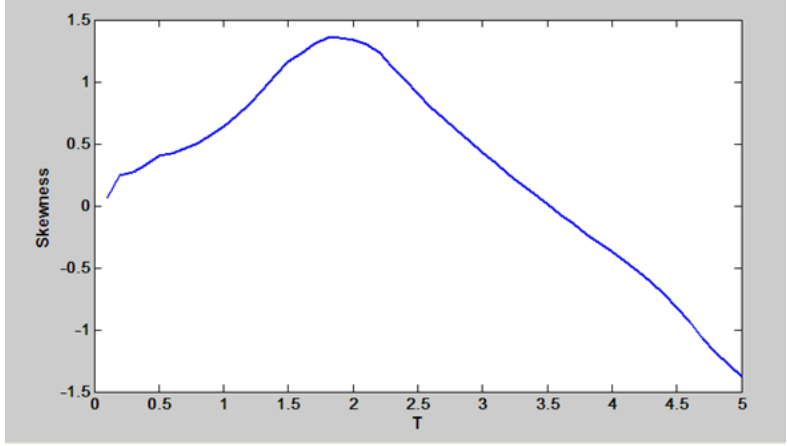

Figure 23. mid-term skewness of SHSZ 300

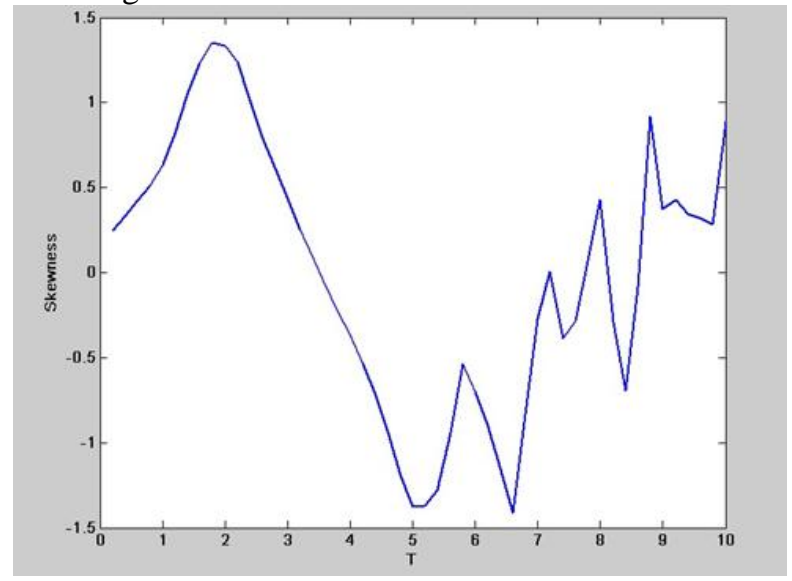

Figure 24. long-term skewness of SHSZ 300 
Figure 22 shows that the short team skewness is positive between 0 and 0.6. While the skewness of S\&P and HSI is negative and decreasing gradually, the skewness of SHSZ 300 is always increasing and approximately positive.

Figure 23 for the five year horizon shows that the skewness of SHSZ 300 starts to decrease after $T=2$, and while $T=3.5$, the skewness becomes negative. Figure 24 for the ten year horizon is zigzagged and gradually increased after $T=5$.

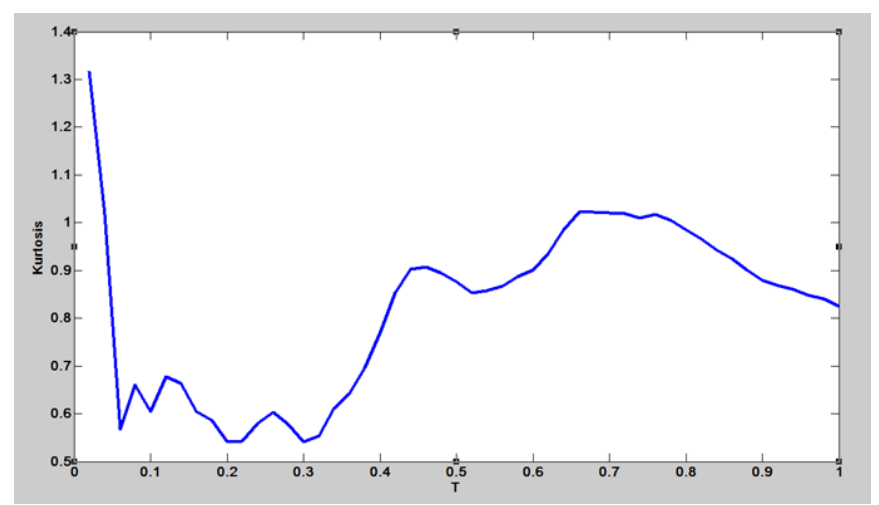

Figure 25. short-term kurtosis of SHSZ 300

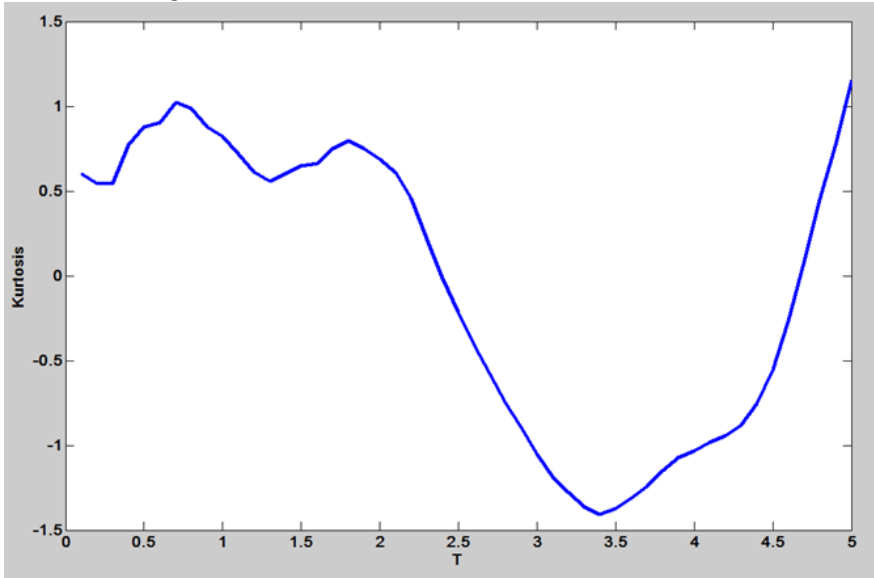

Figure 26. mid-term kurtosis of SHSZ 300

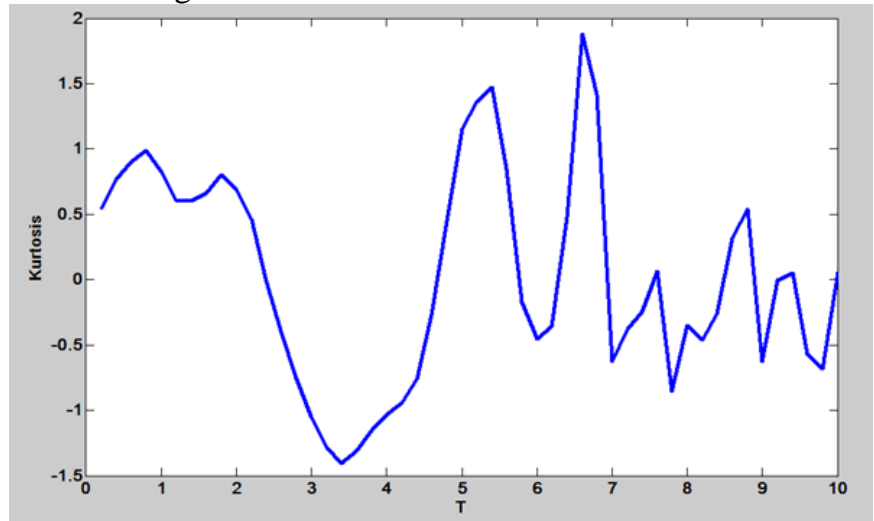

Figure 27. long-term kurtosis of SHSZ 300

Figure 25 shows that for one year horizon the kurtosis changes between 0.5 and 1 . In the same way, there exists no fatter-than-normal tails in the SHSZ 300 return shocks, measured by kurtosis at the confidence level $\alpha=0.01$.

Figure 26 for five year horizon shows that the kurtosis is near to the kurtosis of a normally distributed shock before $T=2.5$. But while $T=3 \sim 4$, the SHSZ 300 return shocks have a little fat tail. Figure 27 for ten year horizon shows that the SHSZ 300 return shocks are approximately near to the kurtosis of a normally distributed shock after $T=4$.

\section{Discussion}

In this paper, we introduce an extension $V a R$ of the traditional VaR for any quantile $\alpha$ level. Our generalized value at risk is a refinement of VaR defined in Engle (2011) for short time horizons, and always makes sense (positive) for long-term horizons. The value at risk VaR defined in Engle (2011) will be negative for some long-term horizons. Our extension VaR provides an possible answer to the challenge question raised by Engle (2011). 
For both S \&P 500, HSI and SHSZ 300, Engle's (2011) VaR always decreases to negative value and our extension VaR always takes positive values and gradually increases to the maximum value 1. In a certain sense, our extension $\operatorname{VaR}$ of the value at risk VaR defined in Engle (2011) provides a risk measure for both short-and long-term risk. From our empirical test on VaR, the extended VaR VaR is more accurate in a short horizon, say 1-year. Hence, it would be possible to apply VaR into Solvency II, QIS, Market Risk and Insurance risks.

With our extended short-and long-term value-at-risk $\mathrm{VaR}$, we introduce the short-and long-term coherent measure of risk (extended expected shortfall) corresponding to the extended value-at-risk. The tail conditional expectation at a level $\alpha$ quantile, and the extended $\alpha$-tail mean as well as the extended expected shortfall are defined and computed (see Proposition 3.1).

\section{Acknowledgements}

This research is supported by the National Natural Science Foundation of China (NO. 71171031, 71471027).

\section{References}

Acerbi, C., \& Tasche, D. (2002). On the coherence of expected shortfall, Journal of Banking and Finance 26, 1487-1503.

Archaya, V., Cooley, T., Richardson, M., \& Walter, I. (2010). Regulating Wall Street: The Dodd-Frank Act and the New Architechture of Global Finance, New York; Wiley Publishing.

Artzner, P., Delbaen, F., Eber, J. -M., \& Heath, D. (1997). Thinking coherently, Risk, 10, 68-71.

Artzner, P., Delbaen, F., Eber, J. -M., \& Heath, D. (1999). Coherent Measures of Risk, Mathematical Finance, 9 , 203-228.

Berd, A., Engle, R., \& Voronov, A. (2007). The underlying dynamics of credit correlations, Journal of Credit Risk 3, 27-62.

Delbaen, F. (2002). Coherent risk measure on general probability spaces, Advances in Finance and Stochastics, 1-37.

Dubil, R., (2009). A Practitioner's critique of Value-at-Risk Models, The VaR Modeling Handbook edited by Gregoriou, G. N., McGraw-Hill Companies, Chapter, 7, 147-181.

Due, D., \& Pan, J. (1997). An overview of Value at Risk, Journal of Derivatives, 4, 7-49.

Edgidio dos Reles, A. D., Gaspar, R. M., \& Vicente, A. T., (2009). Solvency II: An important case in applied VaR, The VaR Modeling Handbook edited by Gregoriou, G. N., McGraw-Hill Companies, Chapter, 12, 267-294.

Embrechts, P. (2000). Extreme value theory: Potential and limitations as an integranted risk management tool, Working paper, ETH Zurich.

Engle, R. (2004). Risk and Volatility: Econometric models and Financial Practice. Noble Lecture. American Economic Review, 94, 405-420.

Engle, R. (2009). The risk that risk will change, Journal of Investment Management, 7, $24-28$.

Engle, R. (2011). Long-term skewness and systemic risk, Journal of Financial Econometrics, 9, 437-468.

Jackson, P., Maude, D. J., \& Perraudin, W. (1997). Bank capital and Value at Risk, Journal of Derivatives.

Marshall, C., \& Sigel, M. (1997). Value at Risk: Implemening a risk measurement standard, Journal of Derivatives.

\section{(cc) BY}

This work is licensed under a Creative Commons Attribution 3.0 License. 